DOI: https://doi.org/10.29105/gmjmx18.34-9

Artículos

\title{
ADAPTACIÓN A WHATSAPP Y RE-ESTANDARIZACIÓN DEL TRABAJO PERIODÍSTICO EN BOLIVIA
}

\section{ADAPTATION TO WHATSAPP AND RE-STANDARDIZATION OF JOURNALISTIC WORK IN BOLIVIA}

\section{Victor Quintanilla-Sangueza}

Universidad Iberoamericana, México

(iD) https://orcid.org/0000-0001-6569-622X

Autor para correspondencia: Victor Quintanilla-Sangueza, email: vico_qs@hotmail.com

\section{Resumen}

Estudios previos acerca del impacto de la innovación tecnológica en el periodismo han tendido a no profundizar lo suficiente en la adaptación de los periodistas al uso de tecnología, lo que ha limitado el análisis de lo que ese cambio ocupacional implica para las condiciones laborales. Esta investigación examina las implicaciones de la innovación tecnológica en el trabajo periodístico a partir de las percepciones y experiencias de periodistas bolivianos en torno al uso de WhatsApp en la recolección de noticias. Bajo una metodología cualitativa, enfatizamos el rol del factor organizacional y el de la agencia profesional en la innovación. Los hallazgos apuntan a que la inclusión rutinaria de esta plataforma de mensajería en prácticas ya estandarizadas de recopilación de noticias deriva en una re-estandarización del trabajo. En esos términos, la innovación implica procesos de adaptación individuales y colectivos en los que el uso de WhatsApp está orientado a lidiar con un ambiente laboral competitivo y responde a exigencias organizacionales, motivaciones individuales y a presiones del contexto. Finalmente, la lógica de conectividad detrás de la plataforma de chat móvil pone a los periodistas en una relación de dependencia con la tecnología que precariza su vida laboral y personal.

Palabras clave: WhatsApp, adaptación profesional, estandarización, periodistas, Bolivia.

\begin{abstract}
Previous studies on the impact of technological innovation in journalism have tended not to look deeply enough into the adaptation of journalists to the use of technology, which has limited the analysis of what this occupational change implies for working conditions. This research examines the implications of technological innovation in journalistic work based on the perceptions and experiences of Bolivian journalists regarding the use of WhatsApp in newsgathering. Under a qualitative methodology, we emphasize the role of the organizational factor and that of professional agency in innovation. The findings suggest that the routine inclusion of this messaging platform in already standardized newsgathering practices leads to a re-standardization of the work. In these terms, innovation
\end{abstract}

Global Media Journal México, 18(35), 176-198, julio - diciembre 2021. 
involves individual and collective adaptation processes in which the use of WhatsApp is oriented to deal with a competitive work environment and responds to organizational demands, individual motivations, and contextual pressures. Finally, the logic of connectivity behind the mobile chat platform puts journalists in a relationship of dependence with technology that makes their work and personal lives precarious.

Keywords: WhatsApp, professional adaptation, standardization, journalists, Bolivia.

Recibido: 31/10/2021

Aceptado: 03/01/2022

\section{Introducción}

Las rutinas de producción de noticias que involucran plataformas de mensajería como WhatsApp son aún poco entendidas (Boczek \& Koppers, 2019). Si bien hay numerosos esfuerzos académicos destinados a entender el impacto de la tecnología en el periodismo, estos tienden a mostrar poco interés en contextos diferentes a los occidentales (Li, 2018) y a no profundizar lo suficiente en los procesos de adaptación que el uso de innovaciones implica para los miembros de una ocupación (Hoff, 2011). La revisión de los estudios que por décadas se han realizado acerca de las rutinas periodísticas sugiere nuevas direcciones de investigación sobre las prácticas e interacciones cada vez más complejas mediante las cuales se distribuye y comparte conocimiento en una suerte de trabajo colaborativo y negociado que involucra a diferentes actores sociales y tecnológicos dentro y fuera de las salas de redacción (Westlund \& Ekström, 2019). Asimismo, un aspecto relevante de la rutinización es que ha sido asociada con la estandarización del trabajo y con una consiguiente descualificación de la fuerza laboral en el periodismo (Reyna García, 2019) y en otras ocupaciones (Leslie \& Rantisi, 2019).

Por otro lado, el contenido noticioso debe entenderse no sólo en función del tipo de información que es de interés periodístico, sino también en el contexto de qué información está disponible para los medios y cómo (Tiffen et al., 2013). Por ello, la recolección de noticias es considerada “el primer eslabón de la cadena de noticias y el núcleo de la actividad periodística” (Reich, 2013, p. 417). El objetivo de este estudio es examinar qué implica para el trabajo periodístico el uso de WhatsApp en la recopilación de noticias. Las tecnologías empleadas en esa etapa pueden configurar la naturaleza del conocimiento que los periodistas obtienen, asociada con aspectos clave de la práctica periodística, entre ellos la confianza en experiencias de primera mano o en las de otras personas, el margen para cuestionar la información brindada por las fuentes o la tendencia a una recepción pasiva (Reich, 2018). El punto de partida son las experiencias y percepciones de 26 periodistas bolivianos. La investigación mantiene así una fuerte apreciación de la agencia de los reporteros 
e indaga al mismo tiempo en el rol de las rutinas de producción de noticias ante la innovación tecnológica. Cabe señalar que el uso de tecnologías como WhatsApp no se limita a la recolección de noticias, sino que atraviesa todo el proceso, siendo, por ejemplo, un nuevo canal de comunicación entre periodistas y audiencias (Boczek \& Koppers, 2019).

Nuestra perspectiva teórica es la de una perspectiva sociológica enfocada en los periodistas y en un entendimiento del periodismo no sólo como profesión, sino también como práctica ocupacional. Esa mirada permite concebir a los periodistas como miembros de una comunidad ocupacional distinta y dar cuenta de su forma de trabajo particular y cambiante (Dickinson, 2007). En esos términos, la investigación se nutre de lo teorizado sobre la innovación, la estandarización del trabajo y la adaptación profesional al cambio ocupacional. Desarrollaremos a continuación este andamiaje teórico para luego explicar la metodología empleada -una de corte cualitativo- y su aplicación. Después, se presentarán y discutirán en detalle los principales hallazgos de investigación, así como las conclusiones centrales. Estos resultados arrojan luz acerca de la complejidad de la recolección de noticias, además de abonar al estudio del periodismo como práctica ocupacional y a los estudios sobre periodismo e innovación tecnológica en América Latina.

\section{Una mirada sociológica a la innovación: estandarización y adaptación}

Aunque la sociología ha sido durante mucho tiempo el "telón de fondo" de los estudios sobre periodismo, su rol y énfasis han sido cambiantes (Zelizer, 2004, p. 45). A decir de Dickinson (2007), uno de los enfoques predominantes ha sido el estudio de las influencias que restringen la práctica periodística, el cual se alejó de los trabajadores o dio cuenta de ellos en términos distintos a los de la sociología ocupacional. El autor defiende los beneficios teóricos de una aproximación sociológica centrada en los periodistas que permita entender de mejor manera qué hacen, cómo y por qué. Ese abordaje permite analizar la situación actual del periodismo en el marco de las tendencias mundiales que afectan al resto de las ocupaciones -entre ellas la desregulación de los mercados laborales, el surgimiento de nuevas formas de empleo, la tecnificación del lugar de trabajo y las preocupaciones sobre la descualificación de la fuerza laboral-, incorporando lo teorizado al respecto desde la sociología del trabajo y de las ocupaciones (Örnebring, 2009). Esta perspectiva resulta necesaria no sólo para entender mejor el proceso de producción de noticias a la luz de los cambios en las condiciones de trabajo y en los contextos de empleo de la comunidad ocupacional más amplia, sino también para conocer cómo los periodistas, sus fuentes y colegas trabajan juntos para llevar a cabo el periodismo (Dickinson, 2007).

Una de las categorías teóricas que permite dar cuenta de las implicaciones del uso de tecnología 
en la labor periodística, con respecto a la situación más amplia de las ocupaciones, es la estandarización del trabajo. Reyna García (2019) señala que, en el periodismo, la estandarización puede ser entendida como "el establecimiento de una serie de estándares con la intención de producir grandes volúmenes de noticias de manera eficaz y predecible" (p. 5). El autor señala que existe un vínculo estrecho entre la estandarización y la rutinización, siendo la primera el establecimiento del patrón y la segunda, su recreación recurrente. La complejidad de la producción de noticias hace que ambos procesos sean necesarios porque ante la falta de predictibilidad y de patrones de conducta, "los periodistas tendrían que decidir cómo recabar y procesar la información de cada evento y probablemente no se podrían mantener los ritmos de producción” (Reyna García, 2019, p. 12). Por ello, el supuesto de que el periodismo siempre se ha regido por rutinas ha prevalecido como un enfoque relevante de estudio.

Desde la sociología del trabajo, Tuchman (1972) fue la primera en hablar de las rutinas en el ámbito del periodismo, afirmando que un elemento clave de la creación noticiosa es la dependencia de procedimientos rutinarios para "procesar la información llamada noticia, un producto agotable hecho cada día" (p. 662). A decir de Becker \& Vlad (2009), los primeros estudios sobre rutinas no vieron que ese factor variaba de un medio a otro, entre los diferentes trabajadores de los medios o en el tiempo. Consideraban a las rutinas como características que definían el trabajo periodístico. Sin embargo, cuando tecnologías como Internet han alterado la forma en la que las noticias se producen y distribuyen, los autores sugieren investigar la variabilidad en las rutinas para comprender sus orígenes y consecuencias: “[...] el investigador necesita encontrar situaciones en las que las rutinas no se siguen o de alguna otra manera se modifican para comprender las consecuencias [...]" (p. 63).

Por otro lado, la implementación de avances científicos y tecnológicos, así como los consiguientes procesos de estandarización del trabajo, han sido vistos como una causa de desprofesionalización desde la sociología de las profesiones (Toren, 1975). En el ámbito periodístico, el declive profesional también ha sido examinado a la luz de las implicaciones del uso de Internet y de otras innovaciones tecnológicas en la práctica periodística (Blankenship, 2015; Lewis, 2012). Sin embargo, el énfasis ha estado en atribuir el fenómeno al avance tecnológico en sí mismo, sin considerar la relación de éste con procesos de estandarización del trabajo. Aunque no es el único ni el más determinante, el componente tecnológico es parte de la innovación en el periodismo. Cabe hacer referencia entonces a lo teorizado sobre la innovación tecnológica y la adaptación a ella de los miembros de una ocupación.

En el periodismo, el concepto de innovación que puede ser empíricamente observado en medios es aquel que la entiende como el descubrimiento y aprovechamiento de una oportunidad mediante el desarrollo de algo nuevo en el ámbito de los productos, tecnologías, procesos y recursos, así como su implementación a través de prácticas profesionales (García-Avilés et al., 2019). De acuerdo con estos autores, los periodistas se han convertido en agentes de cambio en la industria 
mediática, difundiendo las innovaciones por iniciativa personal. A partir de sus hallazgos, conceptualizan la innovación periodística como "un proceso que incluye factores estructurales y prácticos derivados de las aportaciones de los periodistas de diferentes áreas de la producción de noticias" (p. 13). En términos similares, Steensen (2009) defiende que un entendimiento exhaustivo de la innovación en entornos periodísticos es aquel que la ve como un proceso donde la agencia individual y las estructuras organizacionales interactúan. En la misma línea, se ha concluido que, ante los cambios tecnológicos, las percepciones y reacciones de los periodistas son configuradas en parte por atributos individuales (Powers \& Vera-Zambrano, 2019).

La comprensión de la innovación en esos términos coincide con nuestra intención de poner de relieve la adaptación de los periodistas al uso de tecnología. La influencia que el uso de tecnología ejerce en los periodistas y en su labor ha sido ampliamente examinada. Uno de los argumentos ha sido que el periodismo siempre ha estado en transición y que los periodistas siempre se han inclinado por las innovaciones tecnológicas que hacen que su trabajo sea más rápido y eficaz (Dodds, 2019). Dicho planteamiento asume que "muchos de los desarrollos contemporáneos del periodismo pueden verse como una continuación de la subordinación del periodismo a la tecnología creada por el discurso de la velocidad", entendido como una lógica capitalista de competencia y uso de la tecnología para aumentar la productividad (Örnebring, 2010, p. 68). No obstante, el autor reconoce que la tecnología no es una fuerza en sí misma, sino que es implementada y adaptada según sistemas de valores ya existentes con raíces sociales, económicas y culturales.

Sin negar el peso de la tecnología en las percepciones y prácticas de los periodistas, esta investigación arroja nuevas luces sobre lo concluido por estudios previos en el sentido de que la innovación se traduce en procesos de adaptación a nivel individual y grupal que ayudan a los miembros de una ocupación a disminuir su carga de trabajo, ahorrar tiempo valioso y facilitar su labor (Hoff, 2011). Los mecanismos externos orientados a controlar su campo de práctica no eliminan del todo la autoridad de los profesionales para tomar decisiones porque tienden a conciliarlos con su entorno individual y a realizar cambios en función de sus experiencias (Gür, 2014). Como señalamos, un entendimiento integral de la innovación requiere verla como una interacción entre la agencia individual y las estructuras organizacionales. En el periodismo, esta agencia es "el conjunto de actitudes y comportamientos de los individuos" (Deuze, 2009, p. 82). Del mismo modo, la agencia profesional teorizada en estudios sobre otras ocupacionesconsiste en las acciones individuales y colectivas de un grupo de profesionales, así como en el grado de control alcanzado por éste, el cual depende de un diálogo entre las restricciones estructurales externas y el contexto político económico, en equilibrio con las experiencias de vida individuales y colectivas del grupo (Robinson, 2012).

Finalmente, cabe explicar a qué tipo de tecnologías hacemos referencia. Los estudios sobre las implicaciones del desarrollo tecnológico en la 
práctica periodística, particularmente en la recolección de noticias, aluden al uso de redes sociales y de aplicaciones de mensajería móvil. Ambas tecnologías pueden ser consideradas plataformas de contacto interpersonal (Van Dijck, 2016). Esta investigación se circunscribe al uso de WhatsApp, aplicación que entró al mercado en 2010 con el objetivo declarado de reemplazar al servicio de mensajes cortos (SMS por sus siglas en inglés) con un sistema gratuito libre de publicidad (Bouhnik $\&$ Deshen, 2014). WhatsApp es una aplicación multiplataforma de mensajería instantánea para teléfonos móviles inteligentes que permite a los usuarios enviar y recibir en tiempo real -de forma individual y grupal- mensajes de texto, información de localización, imágenes, vídeo, audio, archivos adjuntos y enlaces a direcciones web (Church \& De Oliveira, 2013). Con el tiempo, la plataforma incorporó otras funciones, entre ellas la grabación de mensajes o notas de voz, la búsqueda de palabras clave en una conversación, la eliminación de mensajes ya enviados, así como las llamadas de voz y videollamadas grupales (Pérez, 2021). El alcance de WhatsApp ha estado en constante ascenso, llegando a gestionar el envío de unos 100,000 millones de mensajes cada día en todo el mundo (Chuc, 2020). Su popularidad se debe en gran parte a tres de sus características: un esquema de operación sencillo y accesible a una variedad de personas de diferentes edades y antecedentes; su funcionamiento en casi todos los tipos de dispositivos y sistemas operativos; y un costo total muy bajo (Bouhnik et al., 2014), el cual es nulo para usuarios individuales.
Existe la noción generalizada de que los medios digitales operan como intermediarios y brindan "un apoyo abierto, neutral, equitativo y progresivo a una actividad" (Gillespie, 2010, p. 352). Sin embargo -en vez de ser plataformas abiertas, neutrales y equitativas de interacción social-, introducen nuevos mecanismos tecnológicocomerciales a la comunicación pública que intensifican las estrategias comerciales de los medios (Van Dijck \& Poell, 2015). Conscientes o no de lo que está detrás de la tecnología que usan, Dodds (2019) plantea que los periodistas no son del todo libres de prescindir de ella debido a que la comunicación móvil omnipresente es la norma, a que no es posible escoger la estructura de la cobertura informativa y a que las herramientas que se deben emplear son determinadas por los nuevos hábitos de consumo de las audiencias. El autor explica que dado que la comunicación móvil hace que las personas sean directamente localizables sin importar el lugar donde se encuentren, los periodistas tienen la gran tentación de llegar siempre a una fuente dependiendo de plataformas como WhatsApp y afrontando secuelas reales, profesionales y personales, si prescinden de esa tecnología.

\section{Metodología}

Con base en sus características, consideramos que la investigación cualitativa resultaba útil para analizar nuestro objeto de estudio de forma inductiva: partir de las percepciones y experiencias cotidianas de los 
periodistas en Bolivia para examinar las implicaciones en su trabajo de la inclusión de WhatsApp en la recolección de noticias. Según Soler (2011), los trabajos cualitativos tienen como objeto de estudio a la sociedad diversa, compleja y en constante evolución (con sus creencias, valores, ideas, etc.); tratan de comprender a las personas a partir de cómo ellas conciben su entorno y a ellas mismas; permiten al investigador situarse próximo al mundo empírico, asegurando un ajuste entre los datos y lo que la gente realmente dice y hace. Ese tipo investigación, señala el autor, permite obtener información que una vez analizada lleva a la comprensión de un hecho de un modo inductivo. En el periodismo, los métodos cualitativos proporcionan información valiosa cuando los investigadores examinan de forma exhaustiva los factores que influyen en los periodistas y en su trabajo (Parmelee, 2013).

Los estudios cualitativos implican el uso de una variedad de material empírico y de prácticas interpretativas para "describir momentos y significados rutinarios y problemáticos en la vida de los individuos" (Denzin \& Lincoln, 2008, p. 4). Uno de los métodos más populares en el diseño de la investigación cualitativa es la entrevista, que proporciona al investigador información exhaustiva sobre las experiencias de los participantes y sus puntos de vista respecto de un tema en particular (Turner, 2010). Ese método fue útil para explorar el campo periodístico en Bolivia con base en las historias personales de los reporteros. De entre la variedad de formas que este método adquiere, se escogió la entrevista semiestructurada, cuya fortaleza consiste en la capacidad del investigador de abordar las mismas áreas generales de información de cada entrevistado, teniendo un mayor enfoque que en el abordaje conversacional y, al mismo tiempo, cierta libertad y adaptabilidad para obtener información del entrevistado (McNamara, n.d.). De la aplicación del muestreo de bola de nieve y del muestreo intencional, la entrevista fue aplicada efectivamente a 26 periodistas de las tres ciudades que conforman el eje central de Bolivia (La Paz, Cochabamba y Santa Cruz). De la muestra total, 20 periodistas fueron entrevistados cara a cara. De esas entrevistas, cinco fueron realizadas entre diciembre de 2018 y enero de 2019 , durante la fase piloto, y 15 en la primera fase del trabajo de campo, en junio de 2019. Las últimas seis entrevistas se hicieron entre mayo y agosto de 2020, durante la segunda fase del trabajo de campo, que coincidió con la crisis sanitaria mundial por la COVID-19. Estas entrevistas fueron telefónicas debido a las restricciones impuestas por la pandemia.

Atendiendo a la importancia de contar con una muestra diversa, además de su ubicación geográfica, se consideró el tipo de medio en el que trabajaban los periodistas, su edad, años de experiencia en el periodismo, asignación de cobertura o news beat, cargo y género (Tabla 1). En cuanto al diseño del instrumento, se elaboró un guion con 16 preguntas básicas como plantilla para las entrevistas. Las preguntas fueron divididas en cinco grupos según su utilidad para el logro de nuestro objetivo de investigación: factores individuales y organizacionales, trabajo diario, la relación periodistas-fuentes, uso de WhatsApp y percepción profesional. 


\section{Tabla 1}

Detalle de la muestra de periodistas entrevistados.

\begin{tabular}{|c|c|c|c|c|c|c|}
\hline No. & $\begin{array}{c}\text { Nombre y } \\
\text { tipo de medio }\end{array}$ & Ciudad & $\begin{array}{l}\text { Edad y } \\
\text { género }\end{array}$ & $\begin{array}{c}\text { Años en el } \\
\text { periodismo }\end{array}$ & $\begin{array}{l}\text { Principales } \\
\text { news beats }\end{array}$ & Cargo \\
\hline 1 & $\begin{array}{l}\text { EFE -agencia de } \\
\text { noticias }\end{array}$ & La Paz & $38(\mathrm{M})$ & 15 años & $\begin{array}{l}\text { Política, economía, } \\
\text { cultura y deportes }\end{array}$ & Periodista \\
\hline 2 & $\begin{array}{l}\text { El Deber-diario } \\
\text { impreso }\end{array}$ & La Paz & $53(\mathrm{H})$ & 27 años & Política & $\begin{array}{l}\text { Periodista/ } \\
\text { corresponsal }\end{array}$ \\
\hline 3 & $\begin{array}{l}\text { Los Tiempos-diario } \\
\text { impreso }\end{array}$ & Cochabamba & $30(\mathrm{M})$ & 5 años & Seguridad & Periodista \\
\hline 4 & $\begin{array}{l}\text { El Día-diario } \\
\text { impreso }\end{array}$ & Santa Cruz & $45(\mathrm{H})$ & 20 años & Policial & Periodista \\
\hline 5 & $\begin{array}{l}\text { El Deber-diario } \\
\text { impreso }\end{array}$ & Santa Cruz & $46(\mathrm{H})$ & 17 años & Economía & Periodista \\
\hline 6 & $\begin{array}{l}\text { Página Siete -portal } \\
\text { de diario impreso }\end{array}$ & $\mathrm{La} \mathrm{Paz}$ & $33(\mathrm{M})$ & 3 años & $\begin{array}{l}\text { Nacional, seguridad, } \\
\text { economía, sociedad, } \\
\text { cultura y deportes }\end{array}$ & Periodista \\
\hline 7 & $\begin{array}{l}\text { La Razón -diario } \\
\text { impreso }\end{array}$ & $\mathrm{LaPaz}$ & $47(\mathrm{H})$ & 10 años & Economía & Periodista \\
\hline 8 & Unitel-televisión & Santa Cruz & $39(\mathrm{H})$ & 12 años & $\begin{array}{l}\text { Economía, política, } \\
\text { sociedad y municipios }\end{array}$ & Periodista \\
\hline 9 & El Deber-radio & Santa Cruz & $40(\mathrm{M})$ & 15 años & $\begin{array}{l}\text { Temas de interés } \\
\text { humano }\end{array}$ & $\begin{array}{l}\text { Periodista } \\
\text { y conductora }\end{array}$ \\
\hline 10 & Bolivisión-televisión & Santa Cruz & $34(\mathrm{H})$ & 10 años & Seguridad & Periodista \\
\hline 11 & $\begin{array}{l}\text { Opinión - portal de } \\
\text { diario impreso }\end{array}$ & Cochabamba & $30(\mathrm{H})$ & 10 años & $\begin{array}{l}\text { Ciudad, país, policial, } \\
\text { deportes y cultura }\end{array}$ & Periodista \\
\hline 12 & $\begin{array}{l}\text { Opinión-diario } \\
\text { impreso }\end{array}$ & Cochabamba & $51(\mathrm{M})$ & 30 años & Ciudad & Editora \\
\hline 13 & $\begin{array}{l}\text { Los Tiempos -diario } \\
\text { impreso }\end{array}$ & Cochabamba & $33(\mathrm{M})$ & 8 años & $\begin{array}{l}\text { Política, nacional, } \\
\text { economía y seguridad }\end{array}$ & Macroeditora \\
\hline 14 & $\begin{array}{l}\text { Los Tiempos -portal } \\
\text { de diario impreso }\end{array}$ & Cochabamba & $29(\mathrm{H})$ & 4 años & $\begin{array}{l}\text { Ciudad, economía } \\
\text { y nacional }\end{array}$ & Periodista \\
\hline 15 & Cadena A -televisión & Cochabamba & $37(\mathrm{H})$ & 10 años & Política & $\begin{array}{l}\text { Periodista y } \\
\text { corresponsal }\end{array}$ \\
\hline 16 & ATB -televisión & $\mathrm{LaPaz}$ & $40(\mathrm{M})$ & 18 años & Política & Periodista \\
\hline 17 & $\begin{array}{l}\text { Página Siete-diario } \\
\text { impreso }\end{array}$ & $\mathrm{La} \mathrm{Paz}$ & $35(\mathrm{M})$ & 7 años & Seguridad & Periodista \\
\hline 18 & $\begin{array}{l}\text { Página Siete -diario } \\
\text { impreso }\end{array}$ & $\mathrm{LaPaz}$ & $26(\mathrm{M})$ & 4 meses & Cultura & Periodista \\
\hline 19 & Metropolitana -radio & $\mathrm{LaPaz}$ & $34(\mathrm{M})$ & 7 años & Seguridad & Periodista \\
\hline 20 & Centro -radio & Cochabamba & $30(\mathrm{H})$ & 7 años & Seguridad & Periodista \\
\hline 21 & $\begin{array}{l}\text { Periodismo Policial - } \\
\text { página de Facebook }\end{array}$ & Santa Cruz & $47(\mathrm{H})$ & 20 años & $\begin{array}{l}\text { Seguridad, política, } \\
\text { nacional }\end{array}$ & Periodista \\
\hline 22 & Metropolitana-radio & $\mathrm{LaPaz}$ & $32(\mathrm{H})$ & 5 años & Política & Periodista \\
\hline 23 & Santa Cruz -radio & Santa Cruz & $37(\mathrm{M})$ & 12 años & $\begin{array}{l}\text { Salud, policial y } \\
\text { municipio }\end{array}$ & Periodista \\
\hline 24 & El Deber-radio & Santa Cruz & $52(\mathrm{H})$ & 10 años & Policial & Periodista \\
\hline 25 & Red UNO -televisión & $\mathrm{La} \mathrm{Paz}$ & $37(\mathrm{M})$ & 8 años & Política & Periodista \\
\hline 26 & Pío XII -radio & Cochabamba & $32(\mathrm{M})$ & 8 años & Municipio y policial & Periodista \\
\hline
\end{tabular}


Así como la entrevista, la observación participante también ha sido empleada en estudios contemporáneos para examinar las implicaciones del uso de nuevas tecnologías, en particular aplicaciones de chat móvil como WhatsApp, en las prácticas de producción de noticias (Dodds, 2019). El método ha contribuido a describir ampliamente el uso social de la tecnología y las relaciones entre periodistas (Schmitz Weiss \& Domingo, 2010). No obstante, el desarrollo de las tecnologías digitales plantea nuevas direcciones para la observación, cuyo campo de investigación no puede continuar delimitado por las tradicionales configuraciones físicas cuando, para gran parte del mundo, "la vida diaria cada vez está más mediada tecnológicamente" (Murthy, 2008, p. 849). Los investigadores necesitan "entender mejor cómo se comunican e interactúan las personas en los entornos digitales", lo que "plantea nuevas exigencias a los métodos de investigación con respecto a los textos y entornos digitales en los que los conceptos y herramientas analíticas convencionales pueden necesitar un replanteamiento" (Jewitt, 2013, p. 259).

Atendiendo a lo anterior, se incluyó en el diseño de investigación la observación participante en un entorno digital, el de WhatsApp, donde periodistas bolivianos interactúan entre sí, con sus fuentes y con otros actores como parte de sus prácticas de recolección de noticias. Se ha demostrado que la observación puede realizarse en línea, teniendo como ventaja para la investigación cualitativa la posibilidad de acceder a personas reacias a comunicarse directamente (Angrosino \& Rosenberg, 2012). En las plataformas digitales, los investigadores pueden observar "invisiblemente" interacciones sociales y obtener datos que de otra manera no están disponibles (Murthy, 2008, p. 845).

La aplicación del método consistió en integrarnos a grupos de WhatsApp creados por periodistas en las tres ciudades y a uno creado por una fuente, la Asamblea Legislativa (con sede en La Paz), para luego observar, como un miembro más de cada grupo, las interacciones que allí ocurrían. Esta elección responde a que la observación en ambientes digitales es más fácil de aplicar en grupos orientados a intereses comunes y donde se abordan temas concretos, circunstancia en la que el investigador puede situarse "en un punto ciego que le permita observar el desarrollo de la actividad comunicativa sin una presencia muy invasiva" (Vela \& Cantamutto, 2016, p. 11). Las unidades de observación fueron las intervenciones, que son las más fáciles de segmentar en conversaciones desarrolladas en WhatsApp porque están marcadas visualmente: cuando un interlocutor decide finalizar una intervención, pulsa un botón para enviarla a los receptores y ésta queda registrada en todos los dispositivos junto con la identificación del usuario y la marca temporal del envío (Alcántara Plá, 2014).

Para construir la muestra de observación, se compilaron las intervenciones realizadas en los cuatro grupos de WhatsApp en la semana del 9 al 15 de marzo de 2020, establecida en ese momento como el primer periodo de análisis, el cual coincidió con el estallido global de la crisis sanitaria por la COVID19 y con la etapa previa a la declaratoria de emergencia sanitaria en Bolivia, contexto que amenazaba con afectar, entre otros aspectos, las 
prácticas periodísticas (incluida la recolección de noticias) en nuestro objeto de estudio. Debido a ese contexto y a la necesidad de no sobrecargar la muestra con un solo ciclo de noticias, se optó por realizar otros dos periodos de observación para que la muestra total incluyera también el durante y el después (la fase de post confinamiento declarada en Bolivia) de la crisis sanitaria. El segundo periodo fue del 3 al 9 de agosto de 2020 y el tercero, del 7 al 13 de septiembre de 2020. Cabe recalcar que, si bien la aplicación de la observación fue intervenida por la pandemia, ésta no fue parte del objetivo central del estudio. Ahora bien, los instrumentos para la observación fueron diarios en los que se registró la información teniendo como criterio principal la multimodalidad, que implica dos niveles de datos: el texto limpio o plano y aquel enriquecido con videos, fotografías y otras formas de fijar las manifestaciones multimediales (Herring, 2015).

Para el análisis de los datos obtenidos en las entrevistas y en la observación, nos apoyamos en el análisis cualitativo de contenido, que "permite verificar la presencia de temas, de palabras o de conceptos en un contenido" (Gómez, 2000, p. 106). Cada entrevista fue transcrita y, siguiendo a Cáceres (2003), se definieron las unidades de registro (preguntas) y las de análisis (respuestas). Luego se desarrolló un esquema de codificación con tres niveles de análisis. En el primero, los datos -los fragmentos más representativos de las respuestas de los entrevistados traducidos en citas textualesfueron sistematizados con base en códigos neutrales o anecdóticos, que capturaron tipos más generales de experiencias y percepciones. En una segunda instancia, con base en los temas recurrentes del nivel anterior, los cuales revelaron la consistencia y repetición de percepciones, identificamos fenómenos. Por último, el conjunto amplio de datos y códigos iniciales resultó en un número menor de categorías, establecidas por inferencia teórica, es decir, teniendo en cuenta las categorías definidas antes para analizar el objeto de estudio desde nuestra perspectiva teórica. La información recabada en la observación, registrada previamente en diarios, fue analizada bajo el mismo esquema de codificación y sirvió para confirmar, contrastar y complementar los datos que emanaron de las entrevistas.

\section{Hallazgos}

En Bolivia, la inclusión del uso rutinario de plataformas digitales, principalmente WhatsApp, en procesos ya estandarizados de recolección de noticias ha resultado en la re-estandarización del trabajo periodístico. Pero son los periodistas quienes deciden por su cuenta adoptar esta tecnología y adaptarla a sus necesidades como un paso necesario para responder a las exigencias organizacionales de sus medios, a motivaciones personales y a presiones del contexto político y social del país. Ello quiere decir que están involucrados activamente en la renovada estandarización. Detallaremos a continuación estos hallazgos. 


\section{Re-estandarización: Adaptación y dependencia}

La evidencia empírica da cuenta que los periodistas que hacen parte de nuestro estudio en Bolivia han incorporado a sus procesos rutinarios de recopilación de noticias el uso de plataformas digitales, principalmente WhatsApp y, con menor intensidad, redes sociales (Facebook, Twitter y LinkedIn). Esas herramientas tienen un rol y un uso diferenciado, además de convivir con canales o métodos tradicionales de obtención de información noticiosa, entre ellos la llamada telefónica, el correo electrónico, el contacto cara a cara y la presencia física en el lugar de los hechos. Al haber sido incorporado en las prácticas rutinarias de recolección de noticias, el uso que este grupo de periodistas hace de las plataformas mencionadas también es rutinario.

En el periodismo, las rutinas responden a la necesidad de las organizaciones noticiosas de controlar el trabajo ante sus recursos limitados y la gran cantidad de información susceptible de convertirse en noticia (Becker \& Vlad, 2009). Cabe recalcar que, en la muestra estudiada, el establecimiento de patrones estandarizados orientado a que los periodistas cumplan con las exigencias organizacionales de sus medios, entre ellas la obtención de ideas o alertas de información que pueden convertirse en noticia (Becker \& Vlad, 2009), no es un fenómeno nuevo, sino uno previo y de larga data. De hecho, en términos generales, la rutinización de la producción de noticias viene siendo estudiada por décadas (Westlund \& Ekström, 2019). Es posible señalar entonces que en un entorno de innovación tecnológica estamos ante una nueva estandarización del trabajo periodístico, recreada por nuevas rutinas que prueban que el factor organizacional aún es relevante en la configuración de cómo las noticias son recolectadas. En estas nuevas condiciones, el uso de tecnología puede marcar el inicio de la jornada laboral de los periodistas:

(...) te levantas, alzas tu celular y revisas tu muro, empiezas a revisar Twitter principalmente y a veces sacas ideas de ahi, de temas, una pista suelta y ya tienes el dato para poder hacer un tema (...) (MCH, 14 de junio de 2019).

(...) Me despierto a las 6, veo un poco los noticieros y reviso mi WhatsApp porque la fuente le manda a uno todo lo que pasó (...) (LE, 14 de enero de 2019).

Las respuestas dan cuenta no solamente que la inclusión de WhatsApp y algunas redes sociales al trabajo diario comienza temprano, sino también que -a pesar del impulso externo de las exigencias organizacionales- los periodistas deciden emplear esa tecnología por su cuenta, es decir que no la adoptan de manera forzada, sino que la ven como un paso necesario para desarrollar su labor. Así, en el marco de sus decisiones, los periodistas se ven involucrados activamente en la re-estandarización del trabajo periodístico que la innovación tecnológica motiva. Haciendo una diferenciación entre una plataforma y otra, lo encontrado en Bolivia coincide con aquellos estudios que señalan que el uso de aplicaciones de mensajería instantánea entre 
periodistas ha superado a las redes sociales, siendo WhatsApp la plataforma más significativa (Angeluci et al., 2017; Azeema \& Nazuk, 2018; Dodds, 2019; Kumar \& Haneef, 2016; Negreira-Rey et al., 2017). En la recolección de noticias, el rol que la muestra de periodistas bolivianos concede a WhatsApp es predominante frente al otorgado a las redes sociales, aunque cada plataforma sirve a necesidades concretas. La aplicación de chat móvil es empleada principalmente para coordinar con el medio, tener alertas informativas, acceder a información y verificarla, contactar a fuentes y para realizar cobertura a distancia. El uso de Twitter, Facebook y LinkedIn está orientado sobre todo a obtener alertas informativas e información complementaria susceptible de ser incluida en los contenidos noticiosos. Ocasionalmente, esas plataformas son empleadas para contactar a fuentes.

Ahora bien, en términos generales, la utilidad de la aplicación de mensajería se ha traducido al mismo tiempo en que los periodistas dependan en gran medida de la tecnología para su trabajo diario, lo que ha precarizado su vida laboral y personal. Ese es otro de los hallazgos, posible al problematizar la funcionalidad de plataformas digitales como WhatsApp. Los dispositivos móviles -entre ellos los teléfonos móviles- dan un acceso permanente a redes sociales y aplicaciones de comunicación por Internet, así como a una interacción ubicua, funciones que pueden generar comportamientos compulsivos y un involucramiento excesivo con la tecnología (Choi et al., 2015 y Montag et al., 2015, citados en Wegmann et al., 2017). De otro lado, las plataformas de ese tipo operan bajo una lógica que responde, entre otros principios, a la conectividad, "una táctica estratégica que permite efectivamente la conectividad humana a la vez que impulsa la conectividad automatizada" (Van Dijck \& Poell, 2013, p. 8). Así, las dinámicas detrás de los dispositivos móviles y de las plataformas disponibles en ellos han sido asociadas con una dependencia que no es ajena a la realidad de los periodistas en este estudio:

(...) llego al periódico y conecto mi WhatsApp, abro mi correo y Facebook. Siempre tengo que estar con esas tres [plataformas], y a veces Twitter (...) (AV, 24 de junio de 2019).

(...) Un día se murió el WhatsApp. Nos queríamos morir. Estábamos aquí como locos. No sabiamos qué hacer porque no habia forma de comunicarte, de chatear (...) (CM, 20 de junio de 2019).

(...) Mi herramienta más grande es el WhatsApp, es mi boom. Si me desconectas de WhatsApp un dia, quedo ciego (...) (LE, 14 de enero de 2019).

La relación que los periodistas tienen con la plataforma de mensajería parece responder a su necesidad de mantenerse informados. Al facilitar el acceso en tiempo real a información sobre actividades, hechos y conversaciones, el uso de tecnologías como WhatsApp ha sido asociado al estudio de un fenómeno denominado fear of missing 
out (miedo a ausentarse), definido como "la aprensión generalizada de que otros puedan estar teniendo experiencias gratificantes de las que uno está ausente" y caracterizado "por el deseo de estar continuamente conectado con lo que otros están haciendo" (Przybylski et al., 2013, p. 1841). Para quienes sienten ese temor, las plataformas sociales resultan particularmente atractivas, más si -como señalamos- la dinámica propia de la tecnología es la conectividad mecanizada. La necesidad casi obligada de los periodistas de adoptar aquella tecnología que agilice y haga más eficiente la recolección de noticias tiene su correlato en el desvanecimiento de los límites entre su vida laboral y personal. En torno a sus teléfonos móviles y a WhatsApp, los periodistas siempre están trabajando (Dodds, 2019; Mabweazara, 2011). Así lo evidencia el tiempo que periodistas bolivianos permanecen conectados a la plataforma de chat móvil. La observación participante mostró que, en los grupos de WhatsApp creados por periodistas, las intervenciones se extienden por varias horas cada día, desde las seis de la mañana hasta pasada la medianoche, de lunes a viernes. La dinámica puede ser menos extensa en grupos creados por fuentes, donde las intervenciones comienzan más tarde, a las nueve o 10 de la mañana, y terminan a las 11 o 12 de la noche. Los fines de semana y días festivos, la participación en grupos de periodistas es menor, sobre todo porque los reporteros trabajan en equipos reducidos, sólo quienes están de turno o de guardia. Aún en esos días, la pertenencia a estos grupos es importante para el trabajo periodístico. En otro nivel de análisis, discutiremos ahora la formación y características de esos grupos, que operan como espacios colaborativos de trabajo en el marco del uso rutinario de plataformas digitales.

\section{Adaptación grupal ante la competencia}

En la muestra analizada, identificamos la existencia de tres tipos de grupos de WhatsApp: los de cada medio, creados principalmente para la coordinación interna del trabajo diario entre periodistas, editores y jefes; los creados por una determinada fuente de información (generalmente oficial o institucional), y en los que los periodistas son agregados por áreas de cobertura; y los creados por periodistas, que pueden ser de áreas específicas de cobertura, agrupar a varias áreas o responder a criterios de ubicación (ciudad, por ejemplo). El objetivo central de los dos últimos grupos es el intercambio de información noticiosa. Aunque no fue posible conocer en detalle la conformación de los grupos analizados, sí accedimos a información general al respecto a partir de las entrevistas con los administradores de los grupos y con algunos informantes clave. Como mencionamos, la conectividad es uno de los principios básicos de las plataformas digitales (Van Dijck \& Poell, 2013). Pero debido a la naturaleza privada de gran parte de sus contenidos, la lógica de las aplicaciones de chat móvil como WhatsApp tiene otro elemento básico, el aislamiento, referido a las interacciones que ocurren en grupos cerrados y que con el tiempo se aíslan en términos de participantes y contenido. La conectividad y el aislamiento como características estructurales de estas plataformas son la razón de que periodistas las usen para interactuar con sus colegas 
y con sus fuentes, libres de vigilancia, así como para crear grupos de colaboración (Agur, 2019).

Otro hallazgo relevante es que la conformación de los grupos de WhatsApp refleja el complejo entramado organizacional en el que los periodistas se mueven, el cual involucra no sólo a los medios, sino también a las personas e instituciones que les suministran noticias (Ericson et al., 1987, citados en Dickinson, 2007). En este caso, la estructura con la que interactúan los periodistas que hacen parte del estudio en Bolivia incluye a sus colegas -con quienes intercambian alertas informativas-, así como a sus fuentes y al aparato de comunicación institucional de éstas.

El grupo Mekes de la ciudad de La Paz está conformado en su mayoría por periodistas que realizan cobertura de calle $(70 \%)$, por publirrelacionistas de fuentes oficiales $o$ institucionales $(20 \%)$ y por periodistas o comunicadores que no ejercen su profesión (10\%). El nombre del grupo se relaciona con el término "mequetrefes", de acuerdo con un informante. Los periodistas del grupo tienen diferentes asignaciones de cobertura, incluyendo política, sociedad, seguridad e incluso deportes. El grupo NUEVO PERIODISTAS CBBA, de Cochabamba, está integrado únicamente por periodistas $\mathrm{y}$ por comunicadores que se desempeñan como reporteros. En él predominan los periodistas que cubren las áreas política, municipal y social $(80 \%)$. El resto $(20 \%)$ cubre temas de seguridad y economía. Y el grupo PEPOj -sigla de Periodistas Policiales y Judicialesde Santa Cruz está conformado mayoritariamente por periodistas, pero también incluye a publirrelacionistas de fuentes institucionales y a otros actores interesados en la información que ahí se comparte (como bomberos o rescatistas). Aunque al inicio el grupo tenía como miembros sólo a periodistas especializados en temas de seguridad -de ahí el nombre-, después incorporó también a periodistas de otras áreas (ciudad, sociedad, nacional y deportes). Y el grupo de la Asamblea Legislativa, denominado PRENSA LEGISLATIVO, está integrado por los jefes de las oficinas de comunicación del Senado y de la Cámara de Diputados, comunicadores que trabajan en esas oficinas y por periodistas que cubren las áreas política y nacional en La Paz y en otras ciudades del país. Sus miembros incluyen también a los asesores de los asambleístas, entre quienes hay comunicadores y otro tipo de profesionales.

De otro lado, la evidencia empírica apunta a que la creación de estos espacios responde a motivaciones individuales, particularmente la necesidad de los periodistas de sobrellevar, con ayuda de la tecnología, el ambiente competitivo en el que la práctica periodística se desarrolla. El grupo PEPOj de Santa Cruz, por ejemplo, surgió de la competencia entre periodistas que cubren el área de seguridad. Fue creado un año o dos después de Punto Rojo, un grupo similar. "Como obviamente somos personas celosas de nuestro trabajo, yo también creé mi grupo" (HC, 22 de mayo de 2020), cuenta su fundador. Y el grupo NUEVOS PERIODISTAS CBBA, de Cochabamba, nació de la necesidad de cooperación mutua. Fue creado por seis periodistas de tres medios televisivos diferentes. “(...) Vimos la necesidad de apoyarnos y ayudarnos con 
información, incluso con material; después de un tiempo no muy largo, decidimos agregar a otros que también podían ayudarnos (...)" (CM, 20 de junio de 2019), recuerda uno de ellos. Así, el uso de WhatsApp está orientado a cubrir una gran cantidad de hechos noticiosos en un tiempo limitado. Lo encontrado en Bolivia reafirma que los atributos individuales, entre ellos la posición que los periodistas ocupan en el medio (aspecto asociado a sus años de experiencia), determinan en parte cómo reaccionan frente a los cambios tecnológicos (Powers \& Vera-Zambrano, 2019). En la muestra estudiada, una respuesta de los periodistas ante la inclusión de WhatsApp ha sido dirigir su uso a enfrentar la competencia, lo cual incluye un nuevo tipo de trabajo colaborativo entre colegas.

En el caso de las fuentes, la creación de grupos de WhatsApp responde también a una dinámica de competencia, aquella en la que funcionarios de gobierno y otros miembros de élites luchan por convertirse en noticia, esto bajo el entendido de que sus opiniones no son reproducidas automáticamente en los medios y de que su poder puede variar con el paso del tiempo (Schlesinger, 1990). Nuestros hallazgos en Bolivia revelan que, en esa pugna, los grupos de WhatsApp son una herramienta y un canal de comunicación institucional. El grupo PRENSA LEGISLATIVO fue creado por los jefes de las unidades de comunicación de la Cámara de Senadores y de la Cámara de Diputados para promover entre periodistas que cubren temas de política el trabajo de los diferentes actores de la Asamblea Legislativa.
Como vimos, los periodistas pasan varias horas al día en grupos de WhatsApp como parte de sus rutinas de trabajo. En ese sentido, otra consecuencia del uso de la aplicación de mensajería móvil es la sobrecarga de información que los reporteros experimentan:

\section{(...) Pasa una hora y no has podido ver tu celular porque estabas en entrevistas. Lo abres y ves que habrá conferencia de prensa en media hora y estás con el tiempo mínimo porque estabas en otra cosa. Pero también te satura porque es información. Abres, abres, abres [WhatsApp]. Por hora deben ser más o menos unos 200 mensajes (...) (VL, 21 de junio de 2019).}

Sin embargo, frente al bombardeo de información, los periodistas de la muestra analizada en Bolivia han desarrollado mecanismos individuales de adaptación. Han establecido criterios para definir los mensajes de WhatsApp que es prioritario revisar. Entre ellos están los provenientes de su asignación específica de cobertura, los enviados por los administradores de los grupos creados por fuentes y los mensajes relacionados con temas que están cubriendo en el momento. Y, como segunda estrategia, hacen una distinción entre los grupos donde ya existe una selección interna de temas y los que generalmente no comparten información relevante. Lo encontrado sugiere que ante la innovación tecnológica los periodistas aún son capaces de configurar sus condiciones de trabajo y de conciliar las presiones 
externas con su entorno individual para hacer cambios basados en sus experiencias (Gür, 2014).

\section{Las presiones contextuales}

Además de responder a necesidades organizacionales, el uso de WhatsApp entre periodistas bolivianos también es una decisión motivada por las circunstancias, una reacción a presiones del contexto político y social del país. Como dijimos, los periodistas de la muestra investigada siguen criterios estandarizados en la recolección de noticias, lo que incluye la selección de informantes. En esa etapa, privilegian a las fuentes oficiales que conocen -autoridades, funcionarios, parlamentarios, entre otros-, a quienes consideran fuentes "básicas", “obvias” y "primarias". Las voces extraoficiales -de analistas, expertos o ciudadanos, entre otras-son tomadas en cuenta esporádicamente. Ello da cuenta de la rutinización de la relación periodistas-fuentes y de la tendencia de los periodistas a confiar en fuentes centralizadas ligadas al poder económico y político (Manning, 2001). Los criterios seguidos por los periodistas para la selección de fuentes incluyen además los temas que buscan abordar, la coyuntura noticiosa; la accesibilidad, especialidad y pertinencia de las fuentes; así como su disposición a brindar declaraciones grabadas en audio y/o video. Sin embargo, en el terreno de la accesibilidad y disponibilidad, los periodistas de nuestro segmento de estudio han tropezado con un acceso restringido a la información pública como consecuencia de la polarización política, social e incluso mediática que acompañó la administración del expresidente Evo Morales, fenómeno que no terminó con su salida abrupta del poder, sino que continuó durante el periodo de transición política.

Ese contexto se ha caracterizado por fuentes y publirrelacionistas menos accesibles que antes y con quienes la interacción no es frecuente, un incremento en la solicitud de cuestionarios para el envío de información a periodistas, la designación de voceros autorizados para informar y ruedas de prensa en las que no está permitido que los reporteros formulen preguntas. La polarización múltiple ha resultado además en medios que son percibidos como opositores por el gobierno, una razón adicional para el acceso limitado de los periodistas a fuentes oficiales. Ante el cierre de puertas, el uso de WhatsApp representa una oportunidad para que los periodistas contacten a fuentes oficiales evitando la intermediación de sus publirrelacionistas. Dos periodistas comentan sobre su experiencia:

(...) Tienes el teléfono del ministro, le pones un mensaje en WhatsApp y te contesta. Todas las autoridades contestan; no solo ministros, también el alcalde, el gobernador (...) Incluso puedes hacer entrevistas por WhatsApp porque te piden un cuestionario, lo mandas y te responden (...) (MCH, 14 de junio de 2019).

En cantidad, tengo más fuentes telefónicamente hablando, pero tengo cada vez más fuentes en WhatsApp, donde directamente "puenteas" [evitas] al 
comunicador y saltas directo a la fuente (...) (JC, 15 de enero de 2019).

El segundo hallazgo respecto del peso del contexto es que, ante la crisis sanitaria por la pandemia de la COVID-19, los periodistas bolivianos de la muestra estudiada reforzaron el uso de WhatsApp como herramienta para la recolección de noticias a distancia. En la fase más álgida de la pandemia, prácticamente todos los diarios bolivianos dejaron de circular, excepto uno que mantuvo una distribución reducida en supermercados y farmacias (Quenallata, 2020). Algunas redacciones optaron por el teletrabajo, mientras que fotoperiodistas, así como reporteros de radio y televisión, se vieron obligados a incorporar medidas de bioseguridad a sus procesos de recopilación de noticias (García, 2020). Los periodistas entrevistados en la segunda fase del trabajo de campo, que coincidió con la contingencia sanitaria, dieron cuenta de los cambios y del rol de la tecnología:

(...) durante esta época de la pandemia, nosotros ya casi no podemos entrevistar a las autoridades, lo hacemos a través de WhatsApp (...) (RF, 24 de mayo de 2020).

(...) me dio COVID, entonces no podía salir (...) Tuve que llamar a mis fuentes: “ ¿por favor, me puede mandar una grabación de este temita?". Y la fuente me manda su audio por WhatsApp (...) (JR, 18 de agosto de 2020).
(...) hay mucho más riesgo porque ya no piensas solamente en llevar tu agenda de teléfonos y tu celular, sino que tienes que llevar tu barbijo [cubreboca], tu alcohol en gel. Estamos totalmente cubiertos, conservando todas las medidas de bioseguridad y tratando de cuidar la distancia con los entrevistados porque ellos tampoco tienen la facilidad de salir como antes (...) (PC, 3 de agosto de 2020).

El último testimonio evidencia que la emergencia sanitaria, si bien fortaleció el uso de WhatsApp en las prácticas de recolección de noticias, no implicó que los periodistas dejaran del todo de realizar cobertura presencial. Muestra además que la pandemia no sólo trajo cambios a los métodos rutinarios que los periodistas emplean para obtener noticias, sino también una menor disponibilidad de las fuentes a brindar información, al menos en persona.

Por otro lado, a la luz de los tres periodos establecidos para la observación participante de las interacciones de periodistas en grupos de WhatsApp -antes, durante y después de la declaratoria de emergencia sanitaria por la pandemia- encontramos que los cambios en las dinámicas de periodistas y fuentes no se tradujeron necesariamente en un aumento de las interacciones en la plataforma digital. En los cuatro grupos analizados, el número total de intervenciones durante la pandemia $(1,494)$ y en el periodo de post confinamiento $(1,038)$ fue menor al registrado antes de la declaratoria de emergencia sanitaria, cuando fue de 1,700. Tres son las posibles explicaciones para esos resultados. La primera tiene 
que ver con la continuidad de los eventos noticiosos presenciales, que no fueron trasladados de lleno a espacios digitales. Las alertas informativas compartidas en los grupos de WhatsApp dieron cuenta que -en su mayoría- las ruedas de prensa, declaraciones públicas y otros eventos noticiosos programados siguieron realizándose de forma presencial. La segunda explicación, relacionada con la anterior, es que para muchos periodistas la recolección de noticias en persona no se detuvo por completo. La tercera explicación es que la inclusión de WhatsApp en las prácticas de recopilación de noticias no ocurrió con la pandemia. A diferencia de otras tecnologías digitales de comunicación cuyo uso aumentó exponencialmente a raíz de la emergencia sanitaria, la adaptación de periodistas al uso de la plataforma de mensajería móvil se remonta a mucho tiempo antes de que la contingencia fuera declarada.

Aunque no hubo diferencias significativas en la intensidad de uso de grupos de WhatsApp entre uno y otro periodo de análisis, los ciclos noticiosos abordados en las interacciones sí fueron distintos. La pandemia fue el tema dominante antes de la declaratoria de emergencia sanitaria, mientras que durante la contingencia esa coyuntura compartió protagonismo con la transición política. En la fase de post confinamiento, la transición política, incluida la campaña para las elecciones presidenciales, fue la coyuntura noticiosa principal. La ocurrencia y propagación repentina de la pandemia hizo que fuera incorporada en esta investigación solamente de manera parcial, sin opción a examinar a profundidad sus implicaciones en nuestro objeto de estudio.

\section{Conclusiones}

Los hallazgos discutidos en la sección anterior permitieron desarrollar nuestros principales argumentos. El primero es la constatación de que la inclusión del uso rutinario de plataformas digitales, principalmente WhatsApp, en prácticas ya estandarizadas de recolección de noticias ha traído una re-estandarización del trabajo periodístico en la muestra analizada de periodistas en Bolivia. El segundo es que, en esos términos, la innovación tecnológica implica procesos de adaptación individuales y colectivos para periodistas de ese país, lo que se traduce en su involucramiento activo en la renovada estandarización y en las potenciales consecuencias de ésta para su labor.

Aunque estos periodistas deciden por su cuenta qué tecnología usar y cómo hacerlo para sobrellevar su trabajo diario y, entre otras cosas, responder a las exigencias organizacionales de sus medios, la lógica de la "conectividad automatizada" (Van Dijck \& Poell, 2013, p. 8) de plataformas como WhatsApp los pone en una relación de dependencia con la tecnología que escapa a su control y que difumina los límites entre su vida laboral y personal. Aún así, los periodistas no sólo adoptan la tecnología, sino que también la adaptan a sus necesidades individuales. Son capaces de conciliar con las presiones externas de la tecnología, entre ellas la sobrecarga de información generada del uso extendido de grupos de WhatsApp.

Lo discutido en torno a esos espacios evidencia también la compleja estructura 
organizacional con la que interactúan, la cual involucra no sólo a sus medios, sino también a sus colegas, a sus fuentes y a los publirrelacionistas de éstas. Aunque tienen su origen en motivaciones individuales, los grupos de WhatsApp estudiados son una estrategia colectiva mediante la cual periodistas lidian con un ambiente laboral competitivo. De modo similar, son una herramienta en la competencia de las fuentes por convertirse en noticia. Finalmente, la adaptación de periodistas bolivianos al uso de WhatsApp no responde sólo a exigencias organizacionales y a factores individuales, sino también a presiones del contexto político y social del país, probando la relevancia de los factores contextuales en la implementación de innovaciones tecnológicas (Hoff, 2011) y la necesidad de ver a la tecnología como un elemento insertado en realidades particulares y prácticas preexistentes (Örnebring, 2010).

Desde un contexto perteneciente al Sur Global y poco explorado como el boliviano, la evidencia empírica presentada y discutida en este artículo explica cómo un grupo de periodistas se adapta al uso de WhatsApp - un tipo de tecnología que no ha recibido suficiente atención en los estudios sobre periodismo- en la recolección de noticias y arroja luces acerca de cómo opera la agencia profesional en una parte del periodismo boliviano. En ese sentido, aportamos a la comprensión del grado de control obtenido por un grupo de profesionales a partir de sus acciones individuales y colectivas, así como de su interacción con restricciones externas estructurales y contextuales (Robinson, 2012). También fue posible contrarrestar la tendencia a dar por sentado el carácter innovador de la tecnología y su aporte a la mejora del periodismo. Al partir de las experiencias y percepciones de los propios periodistas, dimos con las diferentes maneras en las que la tecnología es adaptada, así como con sus posibles impactos en las condiciones del trabajo periodístico.

Además de resaltar los aportes de este estudio, es necesario reconocer sus limitaciones, derivadas de aspectos que no fue posible profundizar o abordar del todo, pero los cuales representan insumos para futuras investigaciones. Teniendo en cuenta el alcance de la digitalización en todas las etapas de la producción de noticias, la literatura sobre el tema podría beneficiarse de un análisis exhaustivo de las nuevas ecologías derivadas de la innovación tecnológica en la recolección, selección, producción, presentación y distribución de información.

\section{Referencias bibliográficas}

Agur, C. (2019). Insularized connectedness: Mobile chat applications and news production. Media and Communication, 7(1), 179-188. https://doi.org/10.17645/mac.v7i1.1802

Alcántara Plá, M. (2014). Las unidades discursivas en los mensajes instantáneos de wasap. Estudios de Lingüística del Español, 35, 223-242. https://bit.ly/3ewMfFP 
Angeluci, A. C. B., Donato, R., \& Scolari, G. (2017). O WhatsApp como actante: o impacto do aplicativo interativo em redações jornalísticas. Revista Mediação, 19(24). https://bit.ly/3JmJLIf

Angrosino, M., \& Rosenberg, J. (2012). Observations on observation. Continuities and Challenges. In N. Denzin \& Y. Lincoln (Eds.), Collecting and Interpreting Qualitative Materials (pp. 151-175). SAGE Publications.

Azeema, N., \& Nazuk, A. (2018). WhatsApp and Journalism: News Practices of Pakistani Journalists. Science, Technology and Development, 36(4), 249-258. https://bit.ly/3evkCNm

Becker, L. B., \& Vlad, T. (2009). News Organizations and Routines. In K. Wahl-Jorgensen \& T. Hanitzsch (Eds.), The handbook of journalism studies (pp. 59-72). Routledge.

Blankenship, J. C. (2015). Losing their "mojo"? Mobile journalism and the deprofessionalization of television news work. Journalism Practice, 10(8), 1055-1071. https://doi.org/10.1080/17512786.2015.1063080

Boczek, K., \& Koppers, L. (2019). What's New about Whatsapp for News? A Mixed-Method Study on News Outlets' Strategies for Using WhatsApp. Digital Journalism, 8(1), 126-144. https://doi.org/10.1080/21670811.2019.1692685

Bouhnik, D., \& Deshen, M. (2014). WhatsApp goes to school: Mobile instant messaging between teachers and students. Journal of Information Technology Education: Research, 13(1), 217-231. https://bit.ly/3HbfB8M

Cáceres, P. (2003). Análisis cualitativo de contenido: una alternativa metodológica alcanzable. Psicoperspectivas. Individuo y sociedad, 2(1), 53-81. https://bit.ly/3qOmN4k

Chuc, A. (2020, October 30). WhatsApp ahora entrega aproximadamente 100 mil millones de mensajes al día. La Verdad. https://bit.ly/3tfS1nN

Church, K., \& De Oliveira, R. (2013, August 30). What's up with Whatsapp?: Comparing mobile instant messaging behaviors with traditional SMS [Proceedings]. 15th International Conference on HumanComputer Interaction with Mobile Devices and Services, MobileHCI 2013. Múnich, Alemania. https://bit.ly/3FA1M3y

Dickinson, R. (2007). Accomplishing journalism: Towards a revived sociology of a media occupation. Cultural Sociology, 1(2), 189-208. https://doi.org/10.1177/1749975507078187

Denzin, N. K., \& Lincoln, Y. S. (2008). Collecting and interpreting qualitative materials. Sage.

Deuze, M. (2009). Technology and the individual journalist: Agency beyond imitation and change. In B. Zelizer (Ed.), The changing faces of journalism (pp. 83-97). Routledge.

Dodds, T. (2019). Reporting with WhatsApp: Mobile chat applications' impact on journalistic practices. Digital Journalism, 7(6), 725-745. https://doi.org/10.1080/21670811.2019.1592693

García-Avilés, J. A., Carvajal-Prieto, M., Arias-Robles, F., \& De Lara-González, A. (2019). Journalists' views on innovating in the newsroom: Proposing a model of the diffusion of innovations in media outlets. The Journal of Media Innovations, 5(1), 1-16. http://dx.doi.org/10.5617/jomi.v5i1.3968

Global Media Journal México, 18(35), 176-198, julio - diciembre 2021. 
García, M. (2020, May 10). Periodistas ante la difícil tarea de informar en tiempos de la pandemia. Página Siete. https://bit.ly/3z5sNu

Gillespie, T. (2010). The politics of 'platforms'. New media \& society, 12(3), 347-364. https://doi.org/10.1177/1461444809342738

Gómez, M. (2000). Análisis de contenido cualitativo y cuantitativo: Definición, clasificación y metodología. Revista de Ciencias Humanas, 20(1), 103-113. https://bit.ly/32n2inf

Gür, B. S. (2014). Deskilling of teachers: The case of Turkey. Educational Sciences: Theory and Practice, 14(3), 887-904. https://bit.ly/3mDaKFM

Herring, S. (2015). New frontiers in interactive multimodal communication. In A. Georgopoulou \& T. Spilloti (Eds.), The Routledge handbook of language and digital communication (pp. 398-402). Routledge.

Hoff, T. (2011). Deskilling and adaptation among primary care physicians using two work innovations. Health Care Management Review, 36(4), 338-348. https://doi.org/10.1097/HMR.0b013e31821826a1

Jewitt, C. (2013). Multimodal methods for researching digital technologies. In S. Price, C. Jewitt \& B. Brown (Eds.), The Sage handbook of digital technology research (pp. 250-265). Sage.

Kumar, A., \& Haneef, M. S. (2016). Convergence of technologies and journalists: Translation of journalistic practices through ANT perspective. Estudos Em Comunicação, (22), 105-122. https://doi.org/10.20287/ec.n22.a06

Leslie, D., \& Rantisi, N. (2019). Deskilling in cultural industries: Corporatization, standardization and the erosion of creativity at the Cirque du Soleil. Geoforum, 99, 257-266. https://doi.org/10.1016/j.geoforum.2017.09.011

Lewis, S. C. (2012). The tension between professional control and open participation: Journalism and its boundaries. Information, Communication \& Society, 15(6), 836-866. https://doi.org/10.1080/1369118X.2012.674150

Li, K. (2018). Convergence and de-convergence of Chinese journalistic practice in the digital age. Journalism, 19(9-10), 1380-1396. https://doi.org/10.1177/1464884918769463

Mabweazara, H. M. (2011). Between the newsroom and the pub: The mobile phone in the dynamics of everyday mainstream journalism practice in Zimbabwe. Journalism, 12(6), 692-707. https://doi.org/10.1177/1464884911405468

Manning, P. (2001). News and news sources: A critical introduction. Sage.

McNamara, C. (n.d.). General guidelines for conducting interviews. Free Management Library. https://bit.ly/3qsje3y

Murthy, D. (2008). Digital ethnography: An examination of the use of new technologies for social research. Sociology, 42(5), 837-855. https://doi.org/10.1177/0038038508094565

Global Media Journal México, 18(35), 176-198, julio - diciembre 2021. 
Negreira-Rey, M. C., López-García, X., \& Lozano-Aguiar, L. (2017, March 28). Instant Messaging Networks as a New Channel to Spread the News: Use of WhatsApp and Telegram in the Spanish Online Media of Proximity [Conference paper]. World Conference on Information Systems and Technologies. Madeira, Portugal. https://bit.ly/3pzcT7q

Örnebring, H. (2010). Technology and journalism-as-labour: Historical perspectives. Journalism, 11(1), 57-74. https://doi.org/10.1177/1464884909350644

Parmelee, J. H. (2013). Political journalists and Twitter: Influences on norms and practices. Journal of Media Practice, 14(4), 291-305. https://doi.org/10.1386/jmpr.14.4.291_1

Pérez, C. M. (2021). Análisis de la comunicación y la difusión de contenidos en el grupo de WhatsApp "Tránsito Mashca" [Tesis de Licenciatura, Universidad Técnica de Ambato]. Repositorio Institucional. https://bit.ly/3HVtE2D

Powers, M., \& Vera-Zambrano, S. (2019). Endure, Invest, Ignore: How French and American Journalists React to Economic Constraints and Technological Transformations. Journal of Communication, 69(3), 320-343. https://doi.org/10.1093/joc/jqz015

Przybylski, A. K., Murayama, K., DeHaan, C. R., \& Gladwell, V. (2013). Motivational, emotional, and behavioral correlates of fear of missing out. Computers in Human Behavior, 29(4), 1841-1848. https://doi.org/10.1016/j.chb.2013.02.014

Quenallata, R. (2020, April 8). ESPECIAL: Diarios en Bolivia en riesgo por falta de circulación debido a COVID19. Xinhua. https://bit.ly/3et6WlR

Reich, Z. (2013). The impact of technology on news reporting: A longitudinal perspective. Journalism \& Mass Communication Quarterly, 90(3), 417-434. https://doi.org/10.1177/1077699013493789

Reich, Z. (2018). The decline in orally negotiated news: Revisiting (again) the role of technology in reporting. New media \& society, 20(11), 4116-4134. https://doi.org/10.1177/1461444818768064

Reyna García, V. H. (2019). De la estandarización a la descualificación: las consecuencias indeseadas de la modernización del periodismo mexicano. Comunicación y Sociedad, 16(7072), 1-20. https://doi.org/10.32870/cys.v2019i0.7072

Robinson, S. (2012). Constructing teacher agency in response to the constraints of education policy: Adoption and adaptation. Curriculum Journal, 23(2), 231-245. https://doi.org/10.1080/09585176.2012.678702

Schlesinger, P. (1990). Rethinking the sociology of journalism: Source strategies and the limits of media-centrism. In M. Ferguson (Ed.), Public communication: The new imperatives (pp. 61-83). SAGE Publications.

Schmitz Weiss, A., \& Domingo, D. (2010). Innovation processes in online newsrooms as actor-networks and communities of practice. New media \& society, 12(7), 1156-1171. https://doi.org/10.1177/1461444809360400

Global Media Journal México, 18(35), 176-198, julio - diciembre 2021. 
Soler, P. (2011). La investigación cualitativa. Un enfoque integrador. In L. Vilches (Coord.), La investigación en comunicación. Métodos y técnicas en la era digital (pp. 189-233). Gedisa.

Steensen, S. (2009). What's stopping them? Towards a grounded theory of innovation in online journalism. Journalism studies, 10(6), 821-836. https://doi.org/10.1080/14616700902975087

Tiffen, R., Jones, P.K., Rowe, D., Aalberg, T., Coen, S., Curran, J., Hayashi, K., Iyengar, S., Mazzoleni, G., Papathanassopoulos, S., Rojas, H. \& Soroka, S. (2013). Sources in the news: A comparative study. Journalism studies, 15(4), 374-391. https://doi.org/10.1080/1461670X.2013.831239

Toren, N. (1975). Deprofessionalization and its sources: a preliminary analysis. Sociology of work and occupations, 2(4), 323-337. https://doi.org/10.1177/073088847500200402

Tuchman, G. (1972). Objectivity as strategic ritual: An examination of newsmen's notions of objectivity. American Journal of sociology, 77(4), 660-679. https://doi.org/10.1086/225193

Turner, D. W. (2010). Qualitative interview design: A practical guide for novice investigators. The Qualitative Report,15(3), 754-760. https://doi.org/10.46743/2160-3715/2010.1178

Van Dijck, J., \& Poell, T. (2013). Understanding social media logic. Media and communication, 1(1), 2-14. https://doi.org/10.12924/mac2013.01010002

Van Dijck, J., \& Poell, T. (2015). Social Media and the Transformation of Public Space. Social Media + Society, I(2). https://doi.org/10.1177/2056305115622482

Van Dijck, J. (2016). La cultura de la conectividad. Una historia critica de las redes sociales. Siglo XXI.

Vela, C., \& Cantamutto, L. (2016). De participante a observador: el método etnográfico en el análisis de las interacciones digitales de WhatsApp. Tonos Digital, 31, 1-22. https://bit.ly/3z4PE8f

Wegmann, E., Oberst, U., Stodt, B., \& Brand, M. (2017). Online-specific fear of missing out and Internet-use expectancies contribute to symptoms of Internet-communication disorder. Addictive Behaviors Reports, 5, 33-42. https://doi.org/10.1016/j.abrep.2017.04.001

Westlund, O., \& Ekström, M. (2019). News organizations and routines. In K. Wahl-Jorgensen \& T. Hanitzsch (Eds.), Handbook of Journalism Studies. Routledge. https://doi.org/10.4324/9781315167497

Zelizer, B. (2004). Taking Journalism Seriously: News and the Academy. Sage. 